\title{
Sodium valproate inhibits MDA-MB-231 breast cancer cell migration by upregulating NM23H1 expression
}

\author{
G.-F. Li ${ }^{1 *}$, T.-L. Qian ${ }^{2 *}$, G.-S. Li ${ }^{1}$, C.-X. Yang ${ }^{1}$, M. Qin ${ }^{1}$, J. Huang ${ }^{1}$, \\ M. Sun' ${ }^{2}$ and Y.-Q. Han $^{3}$ \\ ${ }^{1}$ Department of Oncology, The Fourth Affiliated Hospital, \\ Guangxi Medical University, Liuzhou, Guangxi, China \\ 2Department of Anesthesiology, Shenzhen Shekou People's Hospital, \\ Shenzhen, Guangdong, China \\ ${ }^{3}$ Department of Anesthesiology, Central Hospital of Jinan, Jinan, \\ Shandong, China \\ *These authors contributed equally to this study. \\ Corresponding author: G.-F. Li \\ E-mail: ligaofeng05@sina.com
}

Genet. Mol. Res. 11 (1): 77-86 (2012)

Received May 5, 2011

Accepted November 26, 2011

Published January 13, 2012

DOI http://dx.doi.org/10.4238/2012.January.13.1

\begin{abstract}
Breast cancer is a common cancer in women, with a highly variable course, from inoffensive to lethal. To find a more effective strategy for its treatment, sodium valproate has been tested as an anti-cancer drug; it is the only clinically available histone deacetylase inhibitor. However, data about the effects of sodium valproate on breast cancer are insufficient in both animals and humans; studies have yielded conflicting conclusions. In particular, little is known about the association between expression of the metastasis suppressor $\mathrm{Nm} 23 \mathrm{HI}$ gene and breast cancer. We hypothesized that sodium valproate regulates NM23H1 expression, and affects migration and/or invasion. We found that sodium valproate at concentrations of 0.8-3.2 mM inhibits migration and modulates $\mathrm{Nm} 23 \mathrm{H} 1$ gene expression in a concentration-dependent manner. Confluent MDA-MB-231 cells were scratched by a micropipette
\end{abstract}


tip after VPA treatment for $24 \mathrm{~h} ; 24 \mathrm{~h}$ later, the scratch was almostly closed in the $0 \mathrm{mM}$ VPA-treated cells, while the $3.2 \mathrm{mM}$ VPA-treated cells migrated the slowest. The cell migration ratio exposed to $0.8,1.6$ and $3.2 \mathrm{mM}$ VPA was about $66.67,30.67$ and $26.67 \%(\mathrm{P}<0.05)$. We also found evidence that sodium valproate upregulates NM23H1 expression, which is a clue to its anti-cancer mode of action. The NM23H1 gene expression was relative fold increased determined by Western blotting at $3.2 \mathrm{mM}$ VPA. Collectively, these observations indicate that sodium valproate has potential for use in breast cancer treatment.

Key words: Sodium valproate; Breast cancer; Migration; NM23H1

\section{INTRODUCTION}

Breast cancer is one of the most common cancers in females, with a highly variable course, from innofensive to lethal. These variations reflect genetic changes, with chromosomal losses, gains and translocations, often differing between individual patients (Swain et al., 2010). Therefore, more effective strategies for the treatment of breast cancer are clearly needed (Adams et al., 2010).

Valproate acid (VPA) is an antiepileptic drug that inhibits histone deacetylase activity, where it also exerts an anticancer effect. Histone deacetylase inhibitors (HDACIs) represent a promising new class of antineoplastic agents that affect proliferation, differentiation, and apoptosis in both solid and hematologic malignancies (Blaheta et al., 2002). In addition, HDACIs can alter the expression of at least one cellular adhesion molecule. Increased levels of histone acetylation lead to relaxation of the chromatin structure, allowing access of transcription factors and increased transcription, while decreased levels of acetylation are associated with repressed transcription. Therefore, inhibition of the function of histone acetylation may affect the regulation of gene expression, cell growth, differentiation, and apoptosis (Duenas-Gonzalez et al., 2008).

Sodium valproate is the only clinically available histone deacetylase inhibitor at present. Although VPA has been shown to reduce cancer proliferation to some extent, data about its effect on breast cancer cells are still insufficient in both animals and humans, and studies have often yielded contrasting results and conflicting conclusions (Dutertre et al., 2010; Adams et al., 2010; Baneshi et al., 2010). The inhibitory effect of VPA may be cancer type-specific in many studies; for example, one study found no difference in invasiveness between treated and untreated bladder cancer cell lines HTB5 and HTB9 (Byun et al., 2009). This suggests that the mechanism of VPA differs between animal and human tissues. Moreover, while much research has focused on the antiproliferative and differentiation properties of HDACIs, comparatively little is known about their effects on cancer cell migration, invasion, and metastasis (Jawed et al., 2007). Therefore, this study focused on the effect of VPA on human breast cancer cell migration, invasion and metastasis.

A number of oncogenes and tumor suppressor genes that participate in tumorigenesis have been identified, one of them being $N m 23$. The $N m 23$ gene was first identified in a murine malignant melanoma cell; it proved to be a metastasis suppressor gene. Secretion of the NM23 protein can be detected in a variety of tumor cell lines. Among the $\mathrm{Nm} 23$ gene family, the $\mathrm{Nm} 23 \mathrm{H} 1$ gene is the most widely studied; reduced expression of its RNA or gene product is associated with higher stage and/or poorer prognosis in several carcinomas. Decreased levels 
of NM23H1 expression have been linked to metastatic potential. Overexpression of NM23H1 has been demonstrated in highly metastatic murine melanoma cell lines and rat mammary adenocarcinoma cells. Therefore, $\mathrm{Nm} 23 \mathrm{hl}$ represents an ideal target to investigate as a potential therapy for breast cancer (Leone et al., 1991; Jin et al., 2009). While little is known about the association between $\mathrm{Nm} 23 \mathrm{hl}$ expression and breast cancer, $N m 23 \mathrm{hl}$ is a metastasis-suppressor gene in some cancers.

Because acetylation of histones is associated with active transcription, whereas deacetylation by HDACs is linked to gene repression, we hypothesized that VPA would regulate NM23H1 expression, and that migration and/or invasion would also be affected. For these reasons, we investigated the effect of VPA on $\mathrm{Nm} 23 \mathrm{hl}$ gene expression in human breast cancer cells (Fortunati et al., 2008).

\section{MATERIAL AND METHODS}

\section{Cell culture}

MDA-MB-231 human breast cancer cells, obtained from the Cell Resource Center of the Shanghai Life Science Institute of the Chinese Academy of Sciences, were cultured in RPMI 1640 medium supplemented with 10\% fetal bovine serum (Life Technologies, Inc., ON, Canada) with $100 \mathrm{U} / \mathrm{mL}$ penicillin, $100 \mu \mathrm{g} / \mathrm{mL}$ streptomycin and $2.5 \mu \mathrm{g} / \mathrm{mL}$ amphotericin, in a humidified atmosphere containing $5 \% \mathrm{CO}_{2}$.

\section{Effect of VPA on MDA-MB-231 cell assays}

The cells were treated with VPA for $24 \mathrm{~h}$ at concentrations of $0,0.8,1.6$, and $3.2 \mathrm{mM}$. The effect of VPA on MDA-MB-231 cell proliferation was then detected by the methylthiazol tetrazolium (MTT) and wound closure assays. The effect of VPA on histone deacetylation and NM23H1expression was assessed by Western blotting.

\section{Nm23h1 short hairpin RNA (shRNA) plasmid construct and transfection}

shRNA specific for Nm23h1 (NM_008074) was designed according to the Ambion Company's guidelines; a pair of oligonucleotides (sense: 5'-CCGGGTCTGAAGTTTCTG CAGGCTTCTCGAGAAGCCTGCAGAAACTTCAGACTTTTT-3') was synthesized, annealed and ligated to the AgeI and EcoRI sites of Pmko (Ambion Co., USA) to get the plasmid Pmko.1-NM23H1. Insertion was confirmed by sequencing. Before transfection, the cells were seeded in 6-well plates, grown to $30 \%$ confluence, and transfected with complexes containing $1 \mu \mathrm{g} \mathrm{Nm} 23 \mathrm{~h} 1$-shRNA plasmid mixed with Lipofectamine 2000 (Invitrogen, Carlsbad, CA, USA), according to manufacturer recommendations, in serum-free medium for $48 \mathrm{~h}$; $\mathrm{NM} 23 \mathrm{H} 1$ expression was analyzed by Western blotting.

\section{pcDNA3.1/Hygro overexpression vectors and transfections}

The full-length cDNA of the $N m 23 h 1$ gene was isolated from human breast cells and amplified by polymerase chain reaction (PCR). The fragment was cloned into 
pcDNA3.1/Hygro mammalian expression vector (Invitrogen) at EcoRV and $X b a \mathrm{I}$ sites. The insert of the plasmid was sequenced using an ALFexpress automated sequencing machine (Amersham Biosciences, Piscataway, NJ, USA). Stable transfection experiments of the vector containing the $N m 23 h 1$ gene and the empty vector were carried out using Lipofectamine 2000. The cells were diluted and placed under selection $24 \mathrm{~h}$ after transfection with RPMI 1640 medium containing $150 \mathrm{mg} / \mathrm{mL}$ hygromycin B (Invitrogen). MDAMB-231 cells were infected with these plasmids at a multiplicity of infection for $48 \mathrm{~h}$, and analyzed by Western blotting.

\section{Effect of NM23H1 expression on MDA-MB-231 cell assays}

The cells were transfected by $N m 23 h 1$ shRNA plasmids and pcDNA3.1/Hygro overexpression vectors, respectively, to knock down or upregulate the NM23H1 expression. Migration and invasion of transfected cells were detected by a wound closure assay; NM23H1 expression was analyzed by Western blotting.

\section{MTT assay}

Cells at a density of $1 \times 10^{4}$ cells $/ \mathrm{mL}$ were plated in a 96-well plate, at $200 \mu \mathrm{L} /$ well. After overnight incubation, cells were treated with VPA at concentrations of $0,0.8,1.6$, or 3.2 $\mathrm{mM}$, and were harvested at $24 \mathrm{~h}$ following treatment. The effect of VPA on MDA-MB-231 cell proliferation was quantified by the MTT assay (Sigma, USA); $20 \mu \mathrm{L}$ MTT solution ( $5 \mathrm{mg}$ / $\mathrm{mL}$ ) was then added to each well to a final concentration of $0.5 \mathrm{mg} / \mathrm{mL}$ per well. Plates were then cultured for $4 \mathrm{~h}$ at $37^{\circ} \mathrm{C}$. The supernatant was removed, and $150 \mu \mathrm{L}$ DMSO was added to each well to dissolve the deposit. Optical density (OD) was read at $570 \mathrm{~nm}$ using a spectrophotometer microplate reader (Labsystems, Finland). The inhibition of islet cells caused by different concentrations of VPA was calculated using the formula: inhibition rate $(\%)=100 \mathrm{x}$ (1 - $\left.\left[\mathrm{OD}_{\text {drug exposure }} / \mathrm{OD}_{\text {control }}\right]\right)$ (Wu et al., 2008).

\section{Wound closure assay}

Approximately 1 x $10^{5}$ MDA-MB-231 cells were plated in each well of a 6-well plate. After a 12-h incubation without serum, cells were treated with VPA, Nm23h1 siRNA, and pcDNA3.1/Hygro overexpression vectors. The cells were allowed to reach full confluency. Monolayers of cells were scratched by scraping with a micropipette tip, rinsed several times with media to remove dislodged cells, and placed back in growth medium. Photographs were taken using a phase-contrast microscope (Olympus, Japan) immediately or at $24 \mathrm{~h}$ after creating the scratches (Liang et al., 2007).

\section{Western blot analysis}

Western blot analysis of MDA-MB-231 cells was performed as follow: $50-\mu \mathrm{g}$ protein samples were loaded on $8 \%$ polyacrylamide gels, and samples were electrophoresed at $100 \mathrm{~V}$ for $2 \mathrm{~h}$ at room temperature. Protein was transferred to nitrocellulose membranes by 
electroblotting. After the blocking of nitrocellulose membranes in 5\% non-fat dry milk for 1 $\mathrm{h}$, acetylated histone $\mathrm{H} 3$ or $\mathrm{NM} 23 \mathrm{H} 1$ protein was detected by incubating at room temperature for $2 \mathrm{~h}$ with a human monoclonal antibody against acetylated histone $\mathrm{H} 3$ or human NM23H1 (Abcam, England) at a 1:1000 dilution. This was followed by incubating this mixture with a rabbit anti-human secondary antibody conjugated with horseradish peroxidase (HRP) (Amersham) at room temperature for $2 \mathrm{~h}$ at a 1:1000 dilution. Bands were visualized by enhanced chemiluminescence (ECL) (Amersham). The same nitrocellulose membrane was stripped and incubated with $\beta$-actin monoclonal antibody (Sigma) at a 1:2000 dilution for $2 \mathrm{~h}$, which acted as a control for loading and blotting.

\section{Statistical analysis}

Statistical and graphic analyses were performed with the SPSS 13.0 software where appropriate. All experiments were performed in triplicate.

\section{RESULTS}

\section{Effect of VPA on MDA-MB-231 cells}

VPA had no effect on cell proliferation (Figure 1A), but significantly inhibited cell migration in a concentration-dependent manner, as determined by wound closure assays. At $24 \mathrm{~h}$ after creating scratches, cells treated with VPA were significantly slower than the control cells treated with no VPA (Figure 1B), and had higher levels of HDAC and NM23H1 expression. After the MDA-MB-231 cells were treated with $0,0.8,1.6$, or $3.2 \mathrm{mM}$ VPA for $24 \mathrm{~h}$, histone and NM23H1 were assessed by Western blotting. As shown in Figure 2, cells exhibited significantly increased levels of HDAC and NM23H1 compared to untreated controls, in a concentration-dependent manner.

\section{NM23H1-shRNA plasmid to knock down $N m 23 h 1$ increased migration in VPA-treated MDA-MB-231 cells}

In our study, Nm23hl-shRNA significantly decreased the expression of NM23H1, as analyzed by Western blotting (Figure 3A). Subsequent to this decreased NM23H1 expression, these MDA-MB-231 cells migrated faster. After treatment with $3.2 \mathrm{mM}$ VPA for $24 \mathrm{~h}$, migration of RNAi-treated cells was faster than that of cells not treated with RNAi (Figure 3B).

\section{VPA-treated MDA-MB-231 cell migration was inhibited after pcDNA3.1/Hygro overexpression vectors upregulated NM23H1}

In our study, pcDNA3.1/Hygro overexpression vectors significantly upregulated NM23H1 expression, as analyzed by Western blotting (Figure 4A); after NM23H1 expression increased, cell migration was significantly slower than that of control cells (Figure 4B). 
A

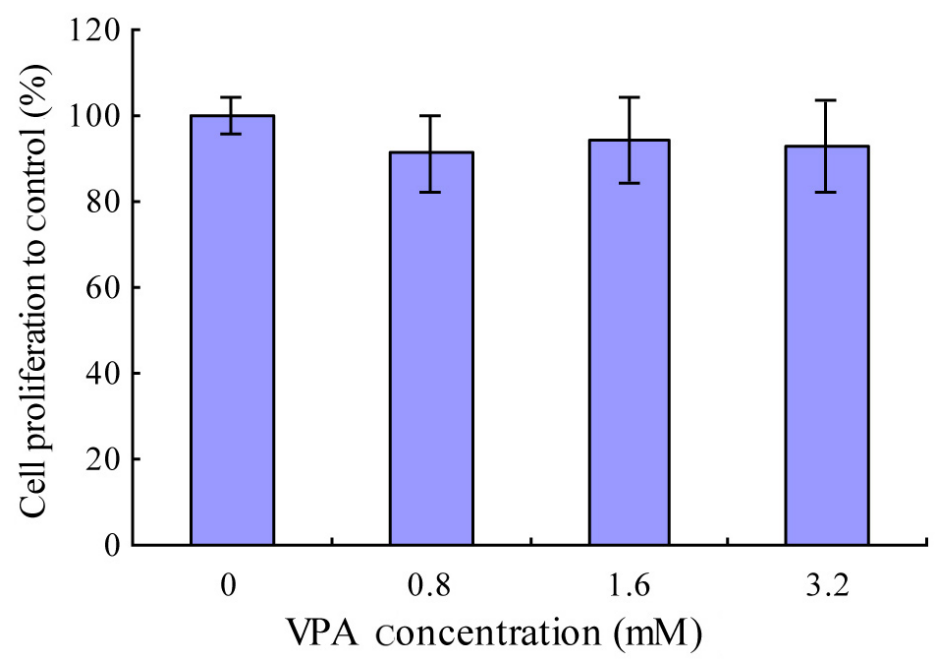

B $\begin{array}{lllll}\mathrm{VPA}(\mathrm{mM}) & 0 & 0.8 & 1.6 & 3.2\end{array}$
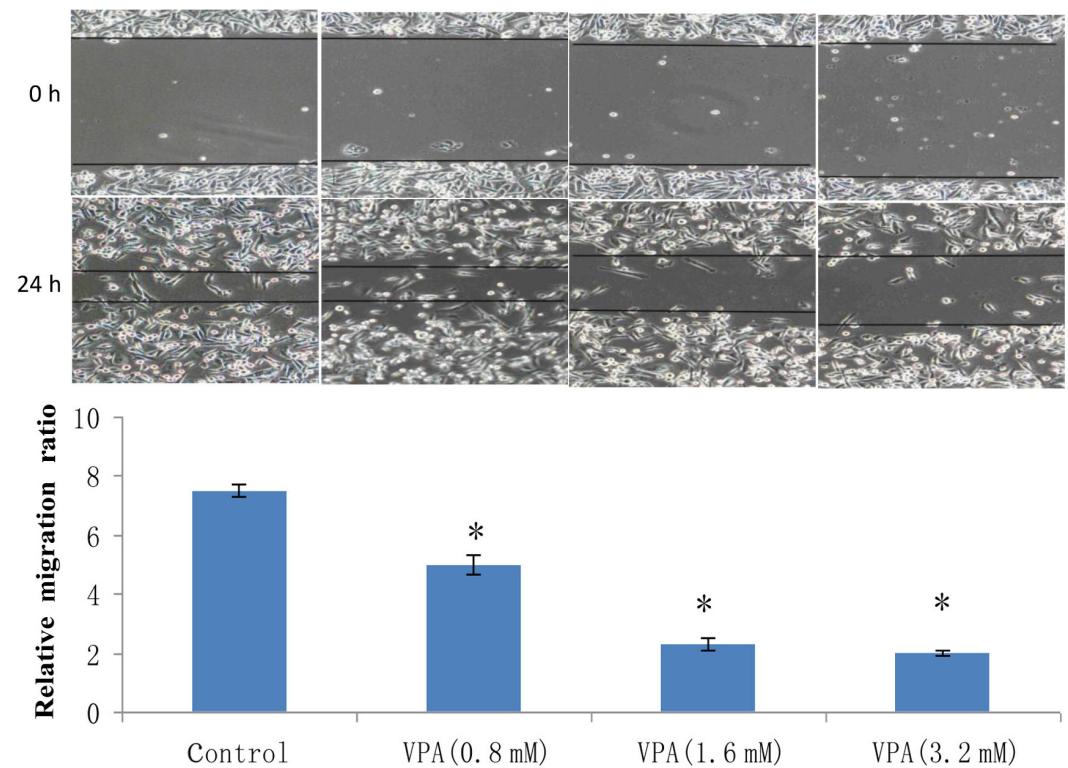

Figure 1. Effect of valproate acid (VPA) on MDA-MB-231 cell proliferation and migration. A. The effect of VPA on cell proliferation quantified by the MTT assay was $100 \%$ in the control group. The proliferation of MDAMB-231 cells exposed to $0.8,1.6$, and 3.2 mM VPA was about 91.2, 94.3 and $92.9 \%$, respectively. There was no difference between groups. The data are reported as means \pm SEM. B. Cell migration at $24 \mathrm{~h}$ was determined by wound closure assays after VPA treatment. VPA inhibited cell migration in a concentration-dependent manner. Confluent MDA-MB-231 cells were scratched by a micropipette tip after VPA treatment for $24 \mathrm{~h} ; 24 \mathrm{~h}$ later, the scratch was almostly closed in the $0 \mathrm{mM}$ VPA-treated cells, while the $3.2 \mathrm{mM}$ VPA-treated cells migrated the slowest. The cell migration ratio exposed to $0.8,1.6$ and $3.2 \mathrm{mM} \mathrm{VPA}$ was about $66.67,30.67$ and $26.67 \%$, respectively, compared with the control cells, which showed a significant difference between groups $(* \mathrm{P}<0.05)$. 
A

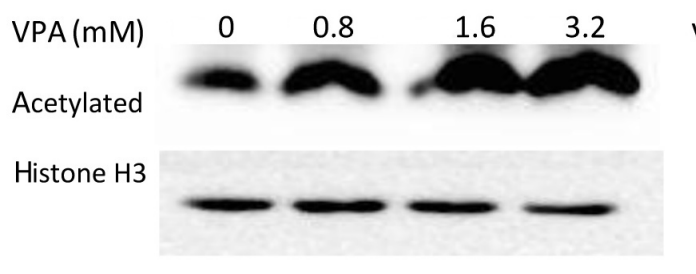

B

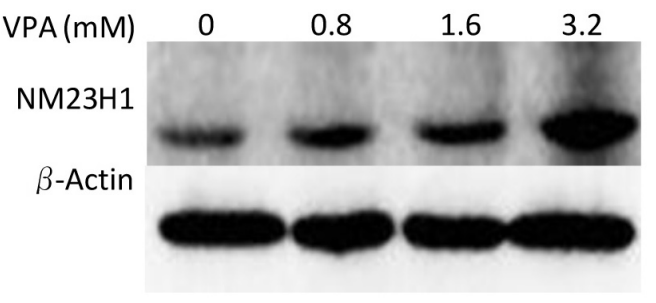

Figure 2. Western blot analysis of acetylated histone H3 and NM23H1 expressions in valproate acid (VPA)treated MDA-MB-231 cells. After the MDA-MB-231 cells were treated with 0, 0.8, 1.6, or $3.2 \mathrm{mM}$ VPA for 24 h, acetylated histone $\mathrm{H} 3$ and NM23H1 were detected by Western blotting. Relative fold increase was determined by scanning densitometry of the Western blots, normalized to $\beta$-actin. VPA treatment resulted in concentrationdependent increases in HDAC and NM23H1 expression.

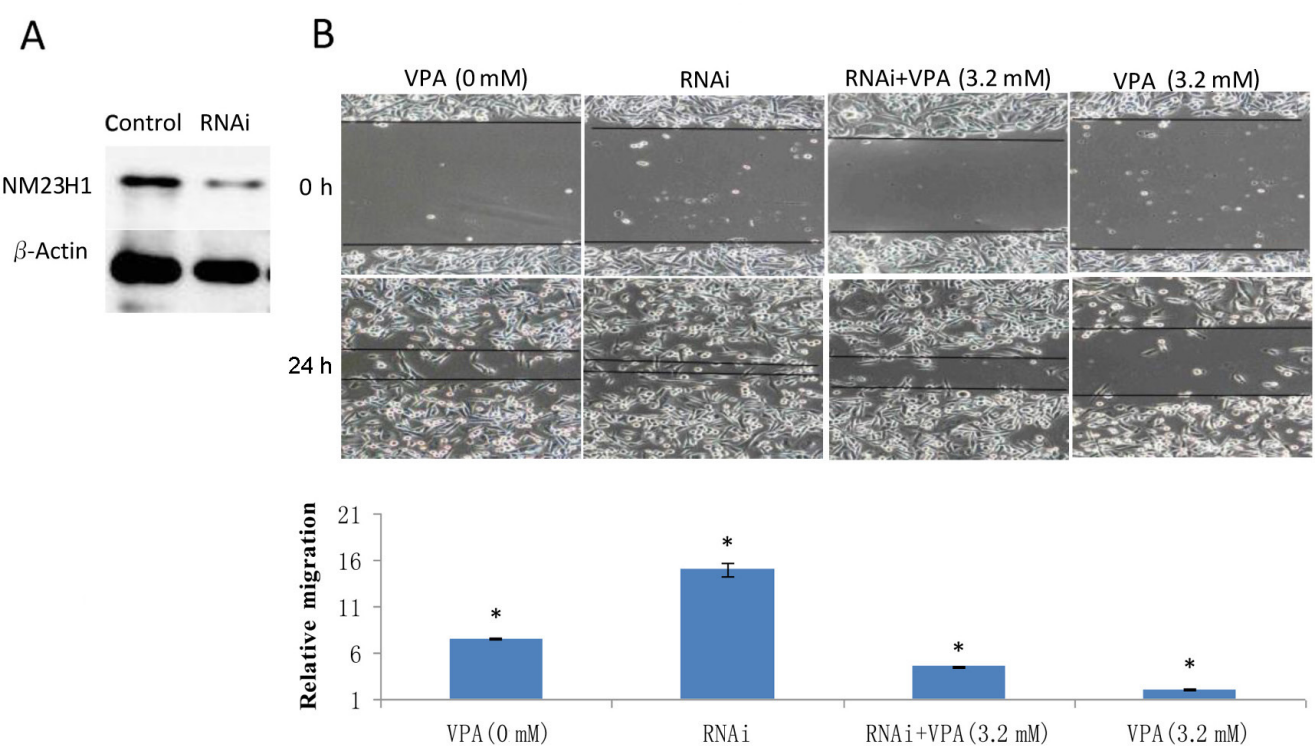

Figure 3. Downregulated NM23H1 expression increases migration in valproate acid (VPA)-treated MDA-MB-231 cells. A. Nm23h1 shRNA knocked down the expression of NM23H1. Whole cell protein lysates from stably transfected cells grown under standard conditions were prepared; expression of NM23H1 in negative-control cells and siRNA cells were analyzed by Western blotting. B. After NM23H1 was knocked dowm by siRNA, cell migration was determined by wound closure assays, and cell migration was significantly faster than in control cells, where the migration ratio increased about $200 \%(\mathrm{P}<0.05)$. Afterwards, the RNAi-treated cells were exposed to $3.2 \mathrm{mM}$ VPA for $24 \mathrm{~h}$; the cell migration was significantly slower, and the migration ratio was reduced about $40 \%$ $(\mathrm{P}<0.05)$. However, in the RNAi-treated cells, migration was significantly faster than in cells without RNAi after exposure to $3.2 \mathrm{mM}$ VPA. $* \mathrm{P}<0.05$ for comparisons between groups. 
A

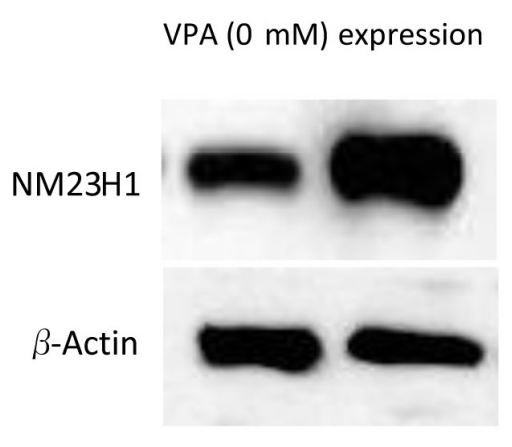

B

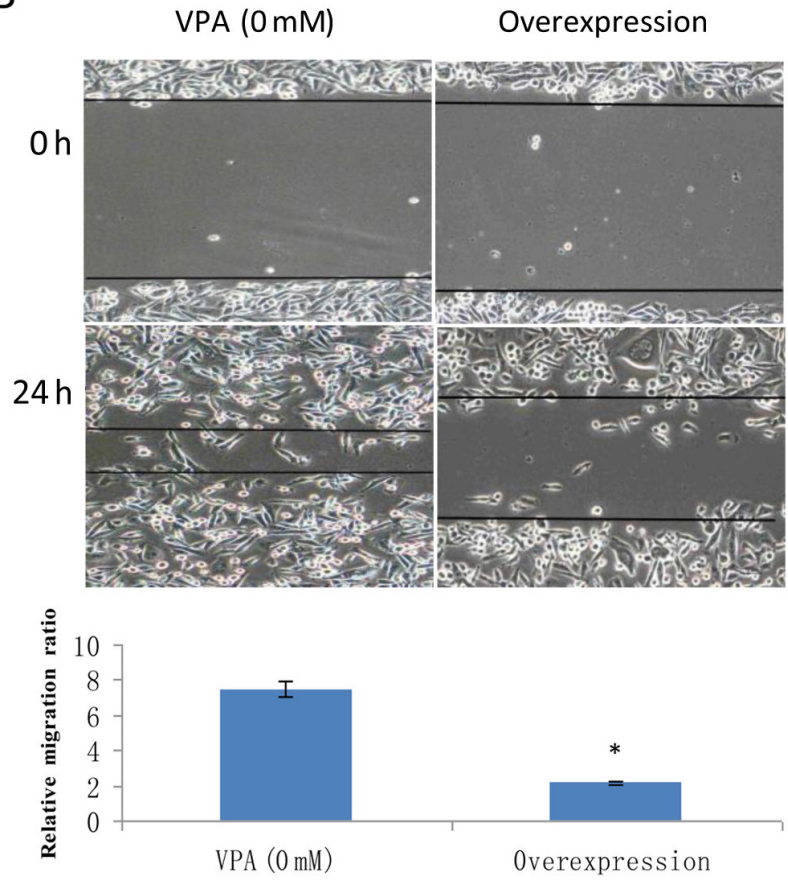

Figure 4. Upregulated NM23H1 expression inhibited valproate acid(VPA)-treated MDA-MB-231 cell migration. A. pcDNA3.1/Hygro overexpression vectors upregulated NM23H1 expression. Whole cell protein lysates from stably transfected cells grown under standard conditions were prepared and analyzed by Western blotting. B. Migration of transfected cells was determined by wound closure assays. Cell migration was significantly inhibited compared to control cells at $24 \mathrm{~h}$ after creating scratches, and the migration ratio was reduced about $70.67 \%(* \mathrm{P}<0.05)$.

\section{DISCUSSION}

HDACIs are a new class of drugs, shown to have antineoplastic activity in hematologic and solid malignancies, which reduce cell proliferation, growth, migration, invasion, and apoptosis and cell-cycle arrest (Lagneaux et al., 2007; Fortunati et al., 2008). Although VPA has been reported to inhibit the proliferation of some cancer cells, it did not do so in the human MDA-MB-231 breast cancer cells in our test, as determined by the MTT assay. We investigated the effects of VPA on human breast cancer cell viability and motility, but at $24 \mathrm{~h}$, we found no acute effects of VPA on cell proliferation in vitro. However, we found that a 24-h treatment with VPA resulted in a concentration-dependent decrease in human MDA-MB-231 cell migration. Our data suggest that VPA exerts some of its antineoplastic effects by inhibiting migration rather than tumor growth. We also found that acute VPA treatment induced concentration-dependent increases in both HDAC and NM23H1, with maximal induction at $3.2 \mathrm{mM}$ for $24 \mathrm{~h}$.

Prior studies of HDACIs have focused on cell-growth inhibition and apoptosis induction as primary mechanisms of their anticancer effects (Dragunow et al., 2006). However, 
newer insights have revealed that HDACI effects on both histone and nonhistone proteins may be more far-reaching than originally thought, modulating cancer cell differentiation, migration and invasion, metastasis, and angiogenesis. Reportedly, HDACIs upregulate a set of metastasis suppressor genes, downregulate a set of metastasis-promoter genes, and repress cancer cell invasion and metastasis in vitro and in vivo (Shen et al., 2005; D'Souza et al., 2009).

We investigated the effect of VPA on the expression of NM23H1, which has a positive role in inhibiting metastasis to explore its anti-cancer mechanism in MDA-MB-231 cells (D'Angelo et al., 2004). Many reports have shown that a loss of NM23H1 expression or function leads to more invasive phenotypes, while restoration of $\mathrm{NM} 23 \mathrm{H} 1$ by conventional means can inhibit invasiveness in many types of tumors (Marshall et al., 2010). The NM23H1 protein may act as an upstream regulator that modulates downstream metastasis-related genes, inhibiting tumor metastasis. However, the mechanism by which NM23H1 participates in tumor metastasis is not fully understood. We supposed that forced overexpression of NM23H1 led to decreased tumor cell proliferation, invasion and metastatic ability, in which $N m 23 \mathrm{hl}$ may be regulated by the HDAC. Downregulation of NM23H1 expression should therefore lead to increased invasiveness and metastasis. This hypothesis is confirmed in our current study. Cell migration was found to be faster after RNAi decreased NM23H1 expression; conversely, cell migration was inhibited after pcDNA3.1/Hygro overexpression vectors increased NM23H1 expression. The mechanism of VPA's anticancer activity therefore seems to involve increased HDAC and NM23H1.

Although apoptosis is reportedly an anticancer mechanism of VPA (Dragunow et al., 2006), we did not evaluate the role of apoptosis in this study. Another limitation of this study is that the VPA treatment in the MDA-MB-231 cells was studied for only $24 \mathrm{~h}$; we cannot assume an effect on cells at 48-72 h, although many studies show that the anticancer effect of VPA improves significantly over longer periods. However, we found clues to the main mechanism of VPA's anti-cancer effect. Additional mechanisms are also possible, such as effects on adhesive function, or even other potential signal pathways (Chen et al., 2007; Platta et al., 2008). Further studies are still required to clarify the exact mechanisms underlying these findings to fully exploit the potential oncostatic benefits of combinatorial therapy with VPA.

In summary, we showed that VPA at concentrations of 0.8-3.2 $\mathrm{mM}$ inhibits migration and modulates NM23H1 expression in a concentration-dependent manner. The concentration range that we studied is achievable therapeutically, and corresponds to serum levels in human. We also present both novel evidence that VPA upregulates NM23H1 expression in human MDA-MB-231 breast cancer cells and clues as to its primary anti-cancer mechanism. All these observations taken together indicate that VPA has a potential role in adjuvant therapies for patients with recurrent, progressive, or muscle-invasive disease, and that it could be the basis of future breast cancer treatments.

\section{REFERENCES}

Adams LS, Phung S, Yee N, Seeram NP, et al. (2010). Blueberry phytochemicals inhibit growth and metastatic potential of MDA-MB-231 breast cancer cells through modulation of the phosphatidylinositol 3-kinase pathway. Cancer Res. 70: $3594-3605$

Baneshi MR, Warner P, Anderson N, Edwards J, et al. (2010). Tamoxifen resistance in early breast cancer: statistical modelling of tissue markers to improve risk prediction. Br. J. Cancer 102: 1503-1510.

Blaheta RA, Nau H, Michaelis M and Cinatl J Jr (2002). Valproate and valproate-analogues: potent tools to fight against cancer. Curr. Med. Chem. 9: 1417-1433. 
Byun SS, Kim FJ, Khandrika L, Kumar B, et al. (2009). Differential effects of valproic acid on growth, proliferation and metastasis in HTB5 and HTB9 bladder cancer cell lines. Cancer Lett. 281: 196-202.

Chen PS, Wang CC, Bortner CD, Peng GS, et al. (2007). Valproic acid and other histone deacetylase inhibitors induce microglial apoptosis and attenuate lipopolysaccharide-induced dopaminergic neurotoxicity. Neuroscience 149: 203212.

D’Angelo A, Garzia L, Andre A, Carotenuto P, et al. (2004). Prune cAMP phosphodiesterase binds nm23-H1 and promotes cancer metastasis. Cancer Cell 5: 137-149.

D'Souza A, Onem E, Patel P, La Gamma EF, et al. (2009). Valproic acid regulates catecholaminergic pathways by concentration-dependent threshold effects on TH mRNA synthesis and degradation. Brain Res. 1247: 1-10.

Dragunow M, Greenwood JM, Cameron RE, Narayan PJ, et al. (2006). Valproic acid induces caspase 3-mediated apoptosis in microglial cells. Neuroscience 140: 1149-1156.

Duenas-Gonzalez A, Candelaria M, Perez-Plascencia C, Perez-Cardenas E, et al. (2008). Valproic acid as epigenetic cancer drug: preclinical, clinical and transcriptional effects on solid tumors. Cancer Treat. Rev. 34: 206-222.

Dutertre M, Gratadou L, Dardenne E, Germann S, et al. (2010). Estrogen regulation and physiopathologic significance of alternative promoters in breast cancer. Cancer Res. 70: 3760-3770.

Fortunati N, Bertino S, Costantino L, Bosco O, et al. (2008). Valproic acid is a selective antiproliferative agent in estrogensensitive breast cancer cells. Cancer Lett. 259: 156-164.

Jawed S, Kim B, Ottenhof T, Brown GM, et al. (2007). Human melatonin MT1 receptor induction by valproic acid and its effects in combination with melatonin on MCF-7 breast cancer cell proliferation. Eur. J. Pharmacol. 560: 17-22.

Jin L, Liu G, Zhang CH, Lu CH, et al. (2009). Nm23-H1 regulates the proliferation and differentiation of the human chronic myeloid leukemia K562 cell line: a functional proteomics study. Life Sci. 84: 458-467.

Lagneaux L, Gillet N, Stamatopoulos B, Delforge A, et al. (2007). Valproic acid induces apoptosis in chronic lymphocytic leukemia cells through activation of the death receptor pathway and potentiates TRAIL response. Exp. Hematol. 35: $1527-1537$.

Leone A, McBride OW, Weston A, Wang MG, et al. (1991). Somatic allelic deletion of nm23 in human cancer. Cancer Res. 51: 2490-2493.

Liang M, Zhang P and Fu J (2007). Up-regulation of LOX-1 expression by TNF-alpha promotes trans-endothelial migration of MDA-MB-231 breast cancer cells. Cancer Lett. 258: 31-37.

Marshall SF, Clarke CA, Deapen D, Henderson K, et al. (2010). Recent breast cancer incidence trends according to hormone therapy use: the California Teachers Study cohort. Breast Cancer Res. 2: R4.

Platta CS, Greenblatt DY, Kunnimalaiyaan M and Chen H (2008). Valproic acid induces Notch1 signaling in small cell lung cancer cells. J Surg. Res. 148: 31-37.

Shen WT, Wong TS, Chung WY, Wong MG, et al. (2005). Valproic acid inhibits growth, induces apoptosis, and modulates apoptosis-regulatory and differentiation gene expression in human thyroid cancer cells. Surgery 138: 979-984.

Swain SM, Jeong JH, Geyer CE Jr, Costantino JP, et al. (2010). Longer therapy, iatrogenic amenorrhea, and survival in early breast cancer. N. Engl. J. Med. 362: 2053-2065.

Wu L, Li Z, Zhang Y, Zhang P, et al. (2008). Adenovirus-expressed human hyperpla- sia suppressor gene induces apoptosis in cancer cells. Mol. Cancer Ther. 7: 222-232. 